\title{
Elevated ASF1B Promotes Apoptosis in Gastric Tumour Cells Through the Bax/Bcl-2-p53 Pathway and is Related to a Good Prognosis
}

\author{
Mengmeng Li \\ Huaihe Hospital of Henan University https://orcid.org/0000-0002-8623-331X \\ Lu Huang \\ Huaihe Hospital of Henan University \\ Yafei Xiao \\ Huaihe Hospital of Henan University \\ Congcong Wang \\ Huaihe Hospital of Henan University \\ Quanying Li \\ Huaihe Hospital of Henan University \\ Changjiang Qin ( $\square$ qincj@vip.henu.edu.cn ) \\ Huaihe Hospital of Henan University https://orcid.org/0000-0003-4295-4964
}

\section{Primary research}

Keywords: ASF1B, gastric tumour, apoptosis, p53, prognosis

Posted Date: September 29th, 2021

DOI: https://doi.org/10.21203/rs.3.rs-929676/v1

License: (a) This work is licensed under a Creative Commons Attribution 4.0 International License. Read Full License 


\section{Abstract}

Background: Previous studies have shown that ASF1B, a H3-H4 histone chaperone, plays a key role in cancer. However, the prognostic relevance and mechanism of ASF1B in patients with gastric cancer (GC) are not clear. Therefore, we explored the prognostic role of ASF1B in gastric cancer and explored its biological function.

Methods: From the bioinformatics perspective, R software was used for prognostic correlation analysis, Gene Set Enrichment Analysis (GSEA) and Weighted Gene Coexpression Network Analysis (WGCNA) were used to screen related differential genes, and R software was used for Gene Oncology $\llbracket G O \bigotimes$ functional enrichment analysis and Kyoto Encyclopedia of Genes and Genomes (KEGG) pathway enrichment analysis. In vitro, CCK-8 colorimetric assays and wound healing assays were used to evaluate the cell proliferation and migration abilities. The mechanism was investigated by western blot and cell functional assays.

Results: Bioinformatics analysis showed that ASF1B was highly expressed in gastric cancer and that its expression was negatively correlated with $\mathrm{T}$ stage and prognosis. Cox regression analysis showed that ASF1B could be used as an independent prognostic factor in gastric cancer. The module genes were identified by WGCNA, and the genes related to ASF1B expression were identified by a PPI coexpression network construction. GO analysis showed enrichment in the terms positive regulation of cell cycle, DNA integrity checkpoint, regulation of double strand break repair, and signal transduction of p53; KEGG analysis showed enrichment in the p53 signalling pathway. GSEA showed that ASF1B was highly enriched in gene sets such as p53 signalling pathway, base excision repair, homologous recombination, and mismatch repair. TIMER analysis showed that the expression of ASF1B was closely related to the key genes of p53 apoptosis. In vitro experiments showed that ASF1B gene knockdown enhanced the proliferation and migration of gastric cancer cells and inhibited gastric cancer cell apoptosis by downregulating P53/Bax and upregulating the expression of the $\mathrm{Bcl}-2$ protein.

Conclusions: ASF1B may be an independent prognostic factor in gastric cancer. Downregulation of ASF1B promotes the proliferation and invasion of gastric tumour cells and is related to poor prognosis.

\section{Introduction}

At present, new cases of gastric cancer (GC) account for $5.7 \%$ of all confirmed cancers, and $8.2 \%$ of all confirmed deaths from cancer are due to gastric cancer. It is the fifth most common type of cancer and the third most fatal cancer worldwide after lung and colorectal cancer, and the vast majority of gastric cancer cases occur in developing countries(1). According to estimates by the International Agency for Research on Cancer (IARC), the gastric cancer had higher morbidity and mortality in 2018 than in 2012(2). Therefore, it is of great importance to find effective targets for the diagnosis and treatment of gastric cancer.

The molecular basis of cancer involves mutations in host genetic factors $(3,4)$. Abnormal gene expression, including gene silencing or overexpression, is associated with DNA methylation variations and abnormal histone posttranslational modifications (5-7). Uncontrolled activity of chromatin regulatory factors, including histone variant proteins, histone chaperones, histone modifying enzymes, effector proteins and chromatin remodelling enzymes, is involved in the occurrence and development of tumours (8-10). Histone $\mathrm{H} 3-\mathrm{H} 4$ chaperone antisilencing function 1 (ASF1) is an important histone chaperone protein that plays an important 
role in cellular DNA replication, DNA damage repair and transcriptional regulation(11). There are two ASF1 subtypes in mammals, ASF1A and ASF1B, both of which act as $\mathrm{H} 3-\mathrm{H} 4$ histone chaperones and provide $\mathrm{N}$ terminal binding interfaces for $\mathrm{H} 3 / \mathrm{H} 4$ histones $(12,13)$. The proteins encoded by ASF1 are substrates of the TLK family of cell cycle regulatory kinases. Chk1 regulates the potential of TLK1 to coordinate ASF1 histone binding and promotes the process of DNA repair $(14,15)$. Related studies have found that the occurrence and prognosis a variety of cancers are closely associated with ASF1B (16-20).

However, to date, there is no relevant literature to demonstrate the possible role of ASF1B in gastric cancer. Therefore, the purpose of this study was to explore the role of ASF1B in gastric carcinoma cells, providing a new direction for future tumour research.

\section{Materials And Methods}

\subsection{Data Acquisition}

We obtained high-throughput sequencing data of GC from the TCGA-STAD dataset (https://xenabrowser.net) (21). Then, with $|\log F C|>1.5$ and $p<0.01$ defined as the threshold criteria for DEGs, we identified DEGs with the limma R package (22). In addition, GEO datasets (GSE84433, GSE29998, GSE13911)

(https://www.ncbi.nlm.nih.gov/geo/query/) were selected to verify the clinical value of ASF1B. Furthermore, the TIMER database (https://cistrome.shinyapps.io/timer) was used to analyse the correlations between ASF1B and key genes in the p53 apoptosis pathway.

\subsection{Weighted Correlation Network Analysis}

WGCNA was used for the construction of a coexpression network, which clustered genes with similar functions into the same module(23). The clusterProfiler R package was applied for $\mathrm{GO}$ enrichment analysis, which included analysis of the molecular functions and biological processes as well as the cellular components of the DEGs, and KEGG) enrichment analysis(24). $P<0.05$ was defined as significant. To further analyse the relationships among proteins, the STRING v11 database (https://string-db.org/) was applied to construct a protein-protein interaction (PPI) network (25).

\subsection{Survival Analysis}

The overall survival of GC patients was investigated by Kaplan-Meier analysis, which was conducted by the survival R package. The KM plotter database (http://www.kmplot.com) is an online database for plotting Kaplan-Meier survival curves from the clinical data in GEO.

\subsection{Gene Set Enrichment Analysis}

GSEA 4.0.3 software was used to analyse the data. GSEA was performed to compare patients with high and low ASF1B expression to study the biological characteristics of our model. To this end, the following cut-off criteria were applied: (i) "collapse dataset to gene symbols" was set as false; (ii) the number of marks was 1000; (iii) the phenotype was set as "permutation type" ; (iv) the "enrichment statistic" was set as weighted; and (v) FDR $<0.25$ and nominal $P$ value $<0.05$ were set as the cut-off criteria. A signal-to-noise metric was used for gene ranking. The high ASF1B group was regarded as the experimental group, while the low ASF1B 
group was defined as the control group. The "c2.cp.kegg.v7.1.symbols.gmt" gene dataset was selected for enrichment analysis.

\subsection{Cell Culture and Small Interfering RNA Transfection}

Human gastric cancer cell lines (MGC803, HGC-27, MKN45, and AGS) were purchased from ATCC (American Type Culture Collection, Manassas, VA, USA). Cells were cultured in RPMI 1640 medium supplemented with $10 \%$ foetal bovine serum (FBS) and $1 \%$ penicillin/streptomycin (streptomycin). All cells were cultured in a $37^{\circ} \mathrm{C}$ humidified incubator containing $5 \% \mathrm{CO}^{2}$.

Small interfering RNA (siRNA) against ASF1B and negative control (NC) RNA were synthesized by Guangzhou RiboBio Company. Cells were seeded in a six-well culture plate and grown to $70 \%$ confluence, and LipofectamineTM 3000 (Invitrogen, Carlsbad, CA, USA) was then used to transfect siRNAs according to the manufacturer's instructions. The siRNA transfection efficiency was determined by qRT-PCR and western blot analysis.

\subsection{Western Blot}

Proteins were separated by SDS-PAGE and transferred to PVDF membranes (Millipore, Billerica, MA, USA). The membranes were blocked in TBST with $5 \%$ skim milk at room temperature for $1 \mathrm{~h}$ and were then incubated with primary antibodies overnight at $4^{\circ} \mathrm{C}$ prior to incubation with rabbit secondary antibodies for 1 h. Enhanced chemiluminescence reagents (CWBIO, Beijing, China) were used to detect antibody binding on autoradiographic films.

\subsection{Proliferation Assay}

The cell proliferation assay was performed by using Cell Counting Kit-8 (CCK-8, Dojindo Molecular Technologies, Rockville, MD, USA) reagent. CRC cells $(100 \mu \mathrm{L})$ at a density of $3.0 \times 10^{5}$ cells/well were seeded in a 96-well plate. Ten microlitres of CCK-8 solution was added to each well at $0 \mathrm{~h}, 24 \mathrm{~h}, 48 \mathrm{~h}$, and $72 \mathrm{~h}$ and cultured at $37^{\circ} \mathrm{C}$ for an additional $4 \mathrm{~h}$. The absorbance at a wavelength of $450 \mathrm{~nm}$ was measured using a microplate reader.

\subsection{Wound Healing Assay}

Transfected cells were seeded into 6-well plates at a density of $3 \times 10^{5}$ cells $/ \mathrm{mL}$ and cultured to $80 \%$ confluence. Then, a linear wound was generated by scraping the cells with a sterile $10 \mu$ pipette tip. Serumfree medium was added to each well after washing with PBS. The migration ability was evaluated by measuring changes in the size of the wound width or area. Images were acquired after $0 \mathrm{~h}$ and $36 \mathrm{~h}$ in three fields of view.

\subsection{Flow Cytometric}

Apoptosis was assessed by Annexin V/propidium iodide (PI) staining followed by flow cytometric (BD Biosciences) analysis according to the manufacturer's instructions. Briefly, harvested cells were washed once with phosphate-buffered saline (PBS) and once with $1 \mathrm{x}$ binding buffer. Next, the cells were incubated with Annexin V-FITC and PI for 15 min at room temperature and were then analysed by flow cytometry. Data analysis was conducted using FlowJo (Tree Star Inc., Ashland, OR) software. 


\subsection{Statistical Analysis}

SPSS20.0 (IBM, USA) was used for analysis. X-tile software was used to determine the best cut-off value. Relationships between ASF1B expression and clinicopathological features were analysed by the chi-square test. Univariate and multivariate Cox regression analyses were performed. Correlations were identified by Pearson correlation analysis. There were significant differences between the two groups $(P<0.05)$.

\section{Results}

\subsection{ASF1B Expression Profiling}

High expression of ASF1B in gastric cancer was verified by GEPIA 2.0 (combined with TCGA and GTEx data). Analysis of TCGA and GEO (GSE29998, GSE13911) datasets also showed that ASF1B was upregulated in gastric cancer $(p<0.001)$. ROC curves showed that ASF1B had very strong sensitivity and specificity for predicting the occurrence of gastric cancer.

\subsection{Clinical Correlation and Prognosis Analysis}

Relevant data were downloaded from TCGA and GEO for clinical correlation analysis. Survival analysis showed that the overall survival and progression-free survival times of patients with gastric cancer with high ASF1B expression were longer than those with low ASF1B expression. By using the "ggpubr" package in R, it was found that the expression of ASF1B was different in different T stages of gastric cancer and was lower in stage T4 than in other stages. Clinical correlation analysis showed that the expression of ASF1B was related to $T$ stage. Univariate and multivariate Cox regression analyses showed that ASF1B could be used as an independent prognostic factor for the survival of patients with gastric cancer.

\subsection{Enrichment Analysis and Construction of PPI}

The gene modules related to ASF1B expression in gastric cancer were identified. According to average linkage analysis and Pearson correlation analysis, 443 samples from patients with gastric cancer were analysed by cluster analysis. Second, the network topology is analysed with various soft thresholding powers; thus, WGCNA has relatively balanced scale independence and average connectivity. After merging modules with a dissimilarity of less than 25\%, 17 different gene modules were identified. Furthermore, the correlation between the MES and ASF1B expression in each gene module was analysed. According to its high correlation with ASF1B expression, the yellow module (Cor $=0.72, p=1.4 \mathrm{e}-128)$ was identified. Then, through the online analysis website STRING, a PPI network was constructed based on the genes in the yellow module. GO and KEGG enrichment analysis of differentially expressed genes in the yellow module showed that ASF1B was enriched in the cell cycle, p53 signalling pathway and foxo pathway. We performed GSEA based on the TCGA cohort. The results showed that the high expression of ASF1B was mainly enriched in the p53 signalling pathway, base excision repair, cell cycle, homologous recombination and mismatch repair gene sets (FDR < 0.25 and NOM P value $<0.05)$.

\subsection{Expression of ASF1B in Gastric Cancer Cell Lines}


Considering that high expression of ASF1B is related to better prognosis, the biological function of ASF1B in gastric cancer cells was studied. By analysing the protein expression of ASF1B in four cell lines by western blotting, we found that HCG27 and MGC803 cells had higher ASF1B expression. Therefore, these two cell lines were selected for follow-up experiments. To further detect the effect of ASF1B on biological behaviours of GC, we used a siRNA transfection technique to silence the expression of ASF1B in HCG27 and MGC803 cells, and western blot analysis was used to determine the transfection efficiency.

\subsection{Knockdown of ASF1B Enhanced the Proliferation and Migration of GC Cells in vitro}

We selected the two gastric cancer cell lines (HCG27 and MGC803) with the highest expression of ASF1B and generated ASF1B-silenced cell lines by siRNA transfection. The CCK-8 assay showed that ASF1B knockout greatly enhanced the proliferation of GC cells. The wound healing assay showed that the migration ability of si-ASF1B cells was significantly enhanced compared with that of the control cells.

\subsection{Knockdown of ASF1B Inhibits Apoptosis through p53 Pathway}

p53 is an important regulator of mitochondrial apoptosis. Flow cytometry was used to explore whether ASF1B affects apoptosis by regulating p53. The results showed that si-ASF1B gastric cancer cells had reduced apoptotic capacity. TIMER analysis showed that ASF1B expression was associated with the expression of the p53, Bax and Bcl-2 genes. Validation of p53-related apoptosis gene protein expression using si-ASF1B transfected HCG-27 and MGC803 gastric cancer cell lines. The western blot results showed that the expression of TP53 was significantly decreased after downregulation of ASF1B, suggesting that p53 may be regulated by ASF1B. At the same time, by detecting the key proteins in the apoptosis-related signalling pathway regulated by $\mathrm{p53}$, it was found that the expression of Bax was downregulated and the expression of bcl-2 was upregulated in the si-ASF1B group compared with the si-NC group. ASF1B may induce apoptosis in gastric cancer cells through the Bax/Bcl-2-p53 axis.

\section{Discussion}

With the improvements in surgical techniques and the progress in traditional radiotherapy and chemotherapy as well as the implementation of neoadjuvant therapy, the 5-year survival rate of early gastric cancer is more than $95 \%$. However, the low rate of early diagnosis means that most patients are diagnosed with advanced disease and therefore miss the best time to undergo surgery (26). Previous studies have shown that gastric cancer biomarkers include HER2, E-cadherin, fibroblast growth factor receptor, mammalian rapamycin target and hepatocyte growth factor receptor, as well as microRNAs, long noncoding RNAs, matrix metalloproteinases, PD-L1, TP53 and microsatellite instability (27). In this study, we found a relationship between ASF1B and gastric cancer, indicating that ASF1B can be used as a new therapeutic target for gastric cancer.

Current studies have shown that ASF1 is a H3-H4 histone chaperone and participates in DNA replication and repair as well as transcriptional regulation $(12,13)$. The prognostic factors in gastric cancer include cell cycle regulatory factors, microsatellite instability (MSI), apoptosis regulatory factors, DNA repair proteins and so 
on(28). The cyclin kinase inhibitor P27KiP1 can slow or even arrest cell division. A study showed that low expression of P27KiP1 is related to poor prognosis in patients with gastric cancer(29). MSI is mainly caused by gene mutation. Recent mutation analysis of gastric cancer shows that there are 37 obviously mutated genes, such as TP53, KRAS, and PIK3, and that the main cause of high mutagenicity is defective DNA mismatch repair $(30,31)$. Similarly, BRCA1 is a tumour suppressor protein that plays an important role in a variety of cellular processes, including DNA damage repair, cell cycle checkpoint control, transcriptional regulation, chromatin remodelling and apoptosis(32-36). The regulation of p53 by BRCA1 can best explain the regulation of transcription factor activity by BRCA1. The interaction between BRCA1 and p53 leads to increased transcription of p53 response promoters, such as p21 and Bax, and induces apoptosis in cancer cells(37).

BRCA1 induces apoptosis by regulating the expression of PIG3 through p53, and the expression of PIG3 is related to better OS in breast cancer patients(38). Because ASF1B and BRCA1 have similar functions in cells and TIMER online analysis showed that BRCA1 expression is highly positively correlated with ASF1B expression, we speculated that ASF1B may play a similar role in gastric cancer. In this study, through bioinformatics multiplatform analysis, it was found that the expression level of ASF1B in gastric cancer tissues was higher than that in normal tissues and that patients with high ASF1B expression had good prognoses, which were related to tumour size and depth of invasion. Cox regression analysis showed that the expression level of ASF1B was an independent risk factor for the prognosis of patients with colorectal cancer. Therefore, we thought that ASF1B may be a molecular marker to predict the development and prognosis of gastric cancer. Then, we investigated the biological function of ASF1B in gastric cancer. Highly expressed genes associated with ASF1B in the module defined by WGCNA were selected for KEGG and GO enrichment analysis, and the results showed that these genes were enriched in the DNA damage repair, cell cycle, transcriptional regulation, chromatin remodelling and p53 signalling pathways. In addition, GSEA of ASF1B was performed, and the results revealed that ASF1B was involved in the above pathways, consistent with our previous speculation. In vitro, an ASF1B knockout cell line was generated. The results showed that low expression of ASF1B promoted the malignant behaviour of gastric cancer by promoting the proliferation and migration of gastric cancer cells.

Activation of the p53 pathway occurs through regulation of apoptosis, cell cycle arrest, differentiation and senescence(39). P53 controls apoptosis through the intrinsic pathway and activates factors that initiate caspase-mediated apoptosis(40). Bcl2 and its family members play an important role in the mitochondrialdependent endogenous apoptotic pathway. Bcl-2 and Bax cooperate to change the mitochondrial membrane potential and permeability, thus initiating the release of regulatory proteins that activate caspases(41). We found that the apoptotic capacity was decreased, the expression of p53 and Bax was inhibited, and the expression of $\mathrm{Bcl}-2$ was upregulated in the cell line with low ASF1B expression, suggesting that ASF1B may exert an antitumour effect by promoting apoptosis through the Bax/Bcl-2-p53 axis.

\section{Conclusions}

Our study shows that high expression of ASF1B indicates good prognosis in gastric cancer and that ASF1B may promote apoptosis through the Bax/Bcl-2-p53 axis to inhibit tumour proliferation. Monitoring the 
expression of ASF1B may provide an important reference for the clinical diagnosis, treatment and prognostic evaluation of gastric cancer.

\section{Declarations}

\section{Conflict of interest}

The authors have declared that no competing interest exists.

\section{Author contributions}

LMM and HL conceived the study protocol. LMM and HL participated in the literature search and the data collection. $\mathrm{LMM}$ and $\mathrm{HL}$ analyzed the data. LMM and $\mathrm{HL}$ drafted the manuscript. $\mathrm{LMM}$ and $\mathrm{HL}$ revised the manuscript. All authors read and approved the final manuscript. LMM and $\mathrm{HL}$ contributed equally to this study.

\section{Funding}

This study was supported by the National Natural Science Foundation of China (NSFC-U1504818), Science and Technology Foundation of Henan Province (172102310152), Natural Science Foundation of Henan Province (182300410359), Science and Technology Foundation of Henan Province (SBGJ202002097), Science and Technology Foundation of Henan Province (192102310099), Henan Provincial Education Fund (19A320020) and Henan University Graduate Program of Excellence (SYL20060192).

\section{Availability of data and materials}

The data sets used and/or analysed during the current study are available from the corresponding author on reasonable request.

\section{Ethics approval and consent to participate}

This study was approved by the Ethics Committee of the Huaihe Hospital of Henan University.

\section{Acknowledgements}

None.

\section{Consent for publication}

We assure that the material is original and it has not been published elsewhere yet. 


\section{References}

1. Bray F, Ferlay J, Soerjomataram I, Siegel RL, Torre LA, Jemal A. Global cancer statistics 2018: GLOBOCAN estimates of incidence and mortality worldwide for 36 cancers in 185 countries. CA Cancer J Clin. 2018;68(6):394-424. doi:10.3322/caac.21492.

2. Thrift AP, El-Serag HB. Burden of Gastric Cancer. Clin Gastroenterol Hepatol. 2020;18(3):534-42. doi:10.1016/j.cgh.2019.07.045.

3. Liu WB, Wu JF, Du Y, Cao GW. Cancer Evolution-Development: experience of hepatitis B virus-induced hepatocarcinogenesis. Curr Oncol. 2016;23(1):e49-56. doi:10.3747/co.23.2836.

4. Gatenby RA, Brown J. Mutations, evolution and the central role of a self-defined fitness function in the initiation and progression of cancer. Biochim Biophys Acta Rev Cancer. 2017;1867(2):162-66. doi:10.1016/j.bbcan.2017.03.005.

5. Abascal F, Corpet A, Gurard-Levin ZA, Juan D, Ochsenbein F, Rico D, et al. Subfunctionalization via adaptive evolution influenced by genomic context: the case of histone chaperones ASF1a and ASF1b. Mol Biol Evol. 2013;30(8):1853-66. doi:10.1093/molbev/mst086.

6. Hattori N, Ushijima T. Compendium of aberrant DNA methylation and histone modifications in cancer. Biochem Biophys Res Commun. 2014;455(1-2):3-9. doi:10.1016/j.bbrc.2014.08.140.

7. Meng CF, Zhu XJ, Peng G, Dai DQ. Promoter histone H3 lysine 9 di-methylation is associated with DNA methylation and aberrant expression of p16 in gastric cancer cells. Oncol Rep. 2009;22(5):1221-7. doi:10.3892/or_00000558.

8. Gurard-Levin ZA, Quivy JP, Almouzni G. Histone chaperones: assisting histone traffic and nucleosome dynamics. Annu Rev Biochem. 2014;83:487-517. doi:10.1146/annurev-biochem-060713-035536.

9. Gurard-Levin ZA, Almouzni G. Histone modifications and a choice of variant: a language that helps the genome express itself. F1000Prime Rep. 2014;6:76. doi:10.12703/P6-76.

10. Avvakumov N, Nourani A, Cote J. Histone chaperones: modulators of chromatin marks. Mol Cell. 2011;41(5):502-14. doi:10.1016/j.molcel.2011.02.013.

11. Peng H, Nogueira ML, Vogel JL, Kristie TM. Transcriptional coactivator HCF-1 couples the histone chaperone Asf1b to HSV-1 DNA replication components. Proc Natl Acad Sci U S A. 2010;107(6):2461-6. doi:10.1073/pnas.0911128107.

12. Paul PK, Rabaglia ME, Wang CY, Stapleton DS, Leng N, Kendziorski C, et al. Histone chaperone ASF1B promotes human beta-cell proliferation via recruitment of histone H3.3. Cell Cycle. 2016;15(23):3191202. doi:10.1080/15384101.2016.1241914.

13. Groth A, Corpet A, Cook AJ, Roche D, Bartek J, Lukas J, et al. Regulation of replication fork progression through histone supply and demand. Science. 2007;318(5858):1928-31. doi:10.1126/science.1148992.

14. Stracker SS-BTH. The Tousled-like kinases regulate genome and epigenome stability: implications in development and disease. Cell Mol Life Sci. 2019;76(19):3827-41.

15. Hammond CM, Stromme CB, Huang H, Patel DJ, Groth A. Histone chaperone networks shaping chromatin function. Nat Rev Mol Cell Biol. 2017;18(3):141-58. doi:10.1038/nrm.2016.159. 
16. Corpet A, De Koning L, Toedling J, Savignoni A, Berger F, Lemaitre C, et al. Asf1b, the necessary Asf1 isoform for proliferation, is predictive of outcome in breast cancer. EMBO J. 2011;30(3):480-93. doi:10.1038/emboj.2010.335.

17. Han G, Zhang X, Liu P, Yu Q, Li Z, Yu Q, et al. Knockdown of anti-silencing function 1B histone chaperone induces cell apoptosis via repressing PI3K/Akt pathway in prostate cancer. Int J Oncol. 2018;53(5):205666. doi:10.3892/ijo.2018.4526.

18. Jiangqiao Z, Tao Q, Zhongbao C, Xiaoxiong M, Long Z, Jilin Z, et al. Anti-silencing function $1 \mathrm{~B}$ histone chaperone promotes cell proliferation and migration via activation of the AKT pathway in clear cell renal cell carcinoma. Biochem Biophys Res Commun. 2019;511(1):165-72. doi:10.1016/j.bbrc.2019.02.060.

19. Rosty C, Sheffer M, Tsafrir D, Stransky N, Tsafrir I, Peter M, et al. Identification of a proliferation gene cluster associated with HPV E6/E7 expression level and viral DNA load in invasive cervical carcinoma. Oncogene. 2005;24(47):7094-104. doi:10.1038/sj.onc.1208854.

20. Ma J, Han W, Lu K. Comprehensive Pan-Cancer Analysis and the Regulatory Mechanism of ASF1B, a Gene Associated With Thyroid Cancer Prognosis in the Tumor Micro-Environment. Frontiers in oncology. 2021;11:711756. doi:10.3389/fonc.2021.711756.

21. Tomczak K, Czerwinska P, Wiznerowicz M. The Cancer Genome Atlas (TCGA): an immeasurable source of knowledge. Contemp Oncol (Pozn). 2015;19(1A):A68-77. doi:10.5114/wo.2014.47136.

22. Ritchie ME, Phipson B, Wu D, Hu Y, Law CW, Shi W, et al. limma powers differential expression analyses for RNA-sequencing and microarray studies. Nucleic Acids Res. 2015;43(7):e47. doi:10.1093/nar/gkv007.

23. Langfelder P, Horvath S. WGCNA: an R package for weighted correlation network analysis. BMC Bioinformatics. 2008;9:559. doi:10.1186/1471-2105-9-559.

24. Yu G, Wang LG, Han Y, He QY. clusterProfiler: an R package for comparing biological themes among gene clusters. OMICS. 2012;16(5):284-7. doi:10.1089/omi.2011.0118.

25. Szklarczyk D, Gable AL, Lyon D, Junge A, Wyder S, Huerta-Cepas J, et al. STRING v11: protein-protein association networks with increased coverage, supporting functional discovery in genome-wide experimental datasets. Nucleic Acids Res. 2019;47(D1):D607-D13. doi:10.1093/nar/gky1131.

26. Song Z, Wu Y, Yang J, Yang D, Fang X. Progress in the treatment of advanced gastric cancer. Tumour Biol. 2017;39(7):1010428317714626. doi:10.1177/1010428317714626.

27. Baniak N, Senger JL, Ahmed S, Kanthan SC, Kanthan R. Gastric biomarkers: a global review. World J Surg Oncol. 2016;14(1):212. doi:10.1186/s12957-016-0969-3.

28. Machlowska J, Maciejewski R, Sitarz R. The Pattern of Signatures in Gastric Cancer Prognosis. Int J Mol Sci (2018) 19(6doi:10.3390/ijms19061658.

29. Nitti D, Belluco C, Mammano E, Marchet A, Ambrosi A, Mencarelli R, et al. Low level of p27(Kip1) protein expression in gastric adenocarcinoma is associated with disease progression and poor outcome. J Surg Oncol. 2002;81(4):167-75. doi:10.1002/jso.10172. discussion 75 - 6.

30. Baretti M, Le DT. DNA mismatch repair in cancer. Pharmacol Ther. 2018;189:45-62. doi:10.1016/j.pharmthera.2018.04.004.

31. Network TCGAR. Comprehensive molecular characterizationof gastric adenocarcinoma. N A T U R E. 2014;513:202-09. doi:10.1038/nature13480. 
32. Huen MS, Sy SM, Chen J. BRCA1 and its toolbox for the maintenance of genome integrity. Nat Rev Mol Cell Biol. 2010;11(2):138-48. doi:10.1038/nrm2831.

33. Marie Cohen1 SP. Christine Wuillemin1, Florence Delie2, Patrick Petignat1 Acellular fraction of ovarian cancer ascites induce apoptosis by activating JNK and inducing BRCA1, Fas and FasL expression in ovarian cancer cells. Oncoscience(2014) 1:262-71.

34. Mullan PB, Quinn JE, Harkin DP. The role of BRCA1 in transcriptional regulation and cell cycle control. Oncogene. 2006;25(43):5854-63. doi:10.1038/sj.onc.1209872.

35. Pathania S, Nguyen J, Hill SJ, Scully R, Adelmant GO, Marto JA, et al. BRCA1 is required for postreplication repair after UV-induced DNA damage. Mol Cell. 2011;44(2):235-51. doi:10.1016/j.molcel.2011.09.002.

36. Zhu Q, Pao GM, Huynh AM, Suh H, Tonnu N, Nederlof PM, et al. BRCA1 tumour suppression occurs via heterochromatin-mediated silencing. Nature. 2011;477(7363):179-84. doi:10.1038/nature10371.

37. Hongbing Zhang3 KS. 2,3, Yi Peng3, Hui Tian1,2,3, Hongxiang Zhang3,Daike Bi3 BLW, 4,5and Wafik S ElDeiry 1,2,3,4,5. BRCA1 physically associates with p53 and stimulates its transcriptional activity. Oncogene. 1998;1998(16):1713-21.

38. Zhang W 1. Jiayan Luo1, *, Fengxia Chen1, *, Fang Yang1, Wei Song2, Aiyu Zhu2, Xiaoxiang Guan. BRCA1 regulates PIG3-mediated apoptosis in a p53-dependent manner. Oncotarget. 2015;6(10):7608-18.

39. Zhang J, Xie Y, Fan Q, Wang C. Effects of karanjin on dimethylhydrazine induced colon carcinoma and aberrant crypt foci are facilitated by alteration of the p53/Bcl2/BAX pathway for apoptosis. Biotech Histochem. 2021;96(3):202-12. doi:10.1080/10520295.2020.1781258.

40. Zhuo Z, Hu J, Yang X, Chen M, Lei X, Deng L, et al. Ailanthone Inhibits Huh7 Cancer Cell Growth via Cell Cycle Arrest and Apoptosis In Vitro and In Vivo. Sci Rep. 2015;5:16185. doi:10.1038/srep16185.

41. Wang JB, Qi LL, Zheng SD, Wu TX. Curcumin induces apoptosis through the mitochondria-mediated apoptotic pathway in HT-29 cells. J Zhejiang Univ Sci B. 2009;10(2):93-102. doi:10.1631/jzus.B0820238.

\section{Tables}


Table 1

Correlation between ASF1B and clinical features in GSE84437 dataset.

\begin{tabular}{|c|c|c|c|c|c|}
\hline \multicolumn{2}{|c|}{ Characteristics } & \multicolumn{4}{|c|}{ ASF1B expression } \\
\hline & & High $(n=320)$ & Low $(n=113)$ & $x^{2}$ & $P$ value \\
\hline \multirow[t]{2}{*}{ Age } & $>60$ & 180 & 59 & 0.551 & 0.458 \\
\hline & $\leq 60$ & 140 & 54 & & \\
\hline \multirow[t]{2}{*}{ Gender } & Male & 218 & 77 & 0.000 & 0.997 \\
\hline & Female & 102 & 36 & & \\
\hline \multirow[t]{4}{*}{ T stage } & T1 & 11 & 0 & 9.863 & $0.020 *$ \\
\hline & T2 & 32 & 6 & & \\
\hline & T3 & 73 & 19 & & \\
\hline & T4 & 204 & 88 & & \\
\hline \multirow[t]{4}{*}{$\mathrm{N}$ stage } & NO & 56 & 24 & 3.694 & 0.296 \\
\hline & N1 & 147 & 41 & & \\
\hline & N2 & 92 & 40 & & \\
\hline & N3 & 25 & 8 & & \\
\hline
\end{tabular}

Table 2

Univariate and multivariate Cox regression analysis of overall survival rate in TCGA dataset.

\begin{tabular}{|lllllllll|}
\hline Characteristics & \multicolumn{3}{l}{ Univariate analysis } & \multicolumn{5}{c|}{ Multivariate analysis } \\
\cline { 2 - 9 } & HR & HR.95L & HR.95H & P value & HR & HR.95L & HR.95H & P value \\
\hline Age & 1.027 & 1.008 & 1.046 & 0.006 & 1.043 & 1.021 & 1.064 & $<0.001$ \\
\hline Gender & 0.674 & 0.445 & 1.021 & 0.062 & & & & \\
\hline Grade & 1.368 & 0.947 & 1.977 & 0.095 & & & & \\
\hline Stage & 1.535 & 1.221 & 1.931 & $<0.001$ & 1.322 & 0.867 & 2.014 & 0.195 \\
\hline T & 1.298 & 1.023 & 1.645 & 0.032 & 1.088 & 0.793 & 1.493 & 0.601 \\
\hline M & 2.048 & 1.096 & 3.827 & 0.025 & 2.037 & 0.908 & 4.570 & 0.084 \\
\hline N & 1.267 & 1.069 & 1.502 & 0.006 & 1.110 & 0.875 & 1.409 & 0.390 \\
\hline ASF1B & 0.973 & 0.949 & 0.998 & $\mathbf{0 . 0 3 2}$ & 0.961 & 0.934 & 0.989 & $\mathbf{0 . 0 0 7}$ \\
\hline
\end{tabular}


Table 3

Univariate and multivariate Cox regression analysis of overall survival rate in GSE84437 dataset.

\begin{tabular}{|lllllllll|}
\hline Characteristics & \multicolumn{3}{l}{ Univariate analysis } & \multicolumn{5}{c|}{ Multivariate analysis } \\
\cline { 2 - 9 } & HR & HR.95L & HR.95H & P value & HR & HR.95L & HR.95H & P value \\
\hline Age & 1.027 & 1.007 & 1.032 & 0.002 & 1.024 & 1.011 & 1.036 & $<0.001$ \\
\hline Gender & 1.256 & 0.928 & 1.700 & 0.141 & & & & \\
\hline T & 1.740 & 1.378 & 2.198 & $<0.001$ & 1.567 & 1.227 & 2.000 & $<0.001$ \\
\hline N & 1.676 & 1.429 & 1.967 & $<0.001$ & 1.532 & 1.303 & 1.409 & $<0.001$ \\
\hline ASF1B & 0.785 & 0.665 & 0.927 & $\mathbf{0 . 0 0 4}$ & 0.805 & 0.679 & 0.954 & $\mathbf{0 . 0 1 2}$ \\
\hline
\end{tabular}

Figures 
A

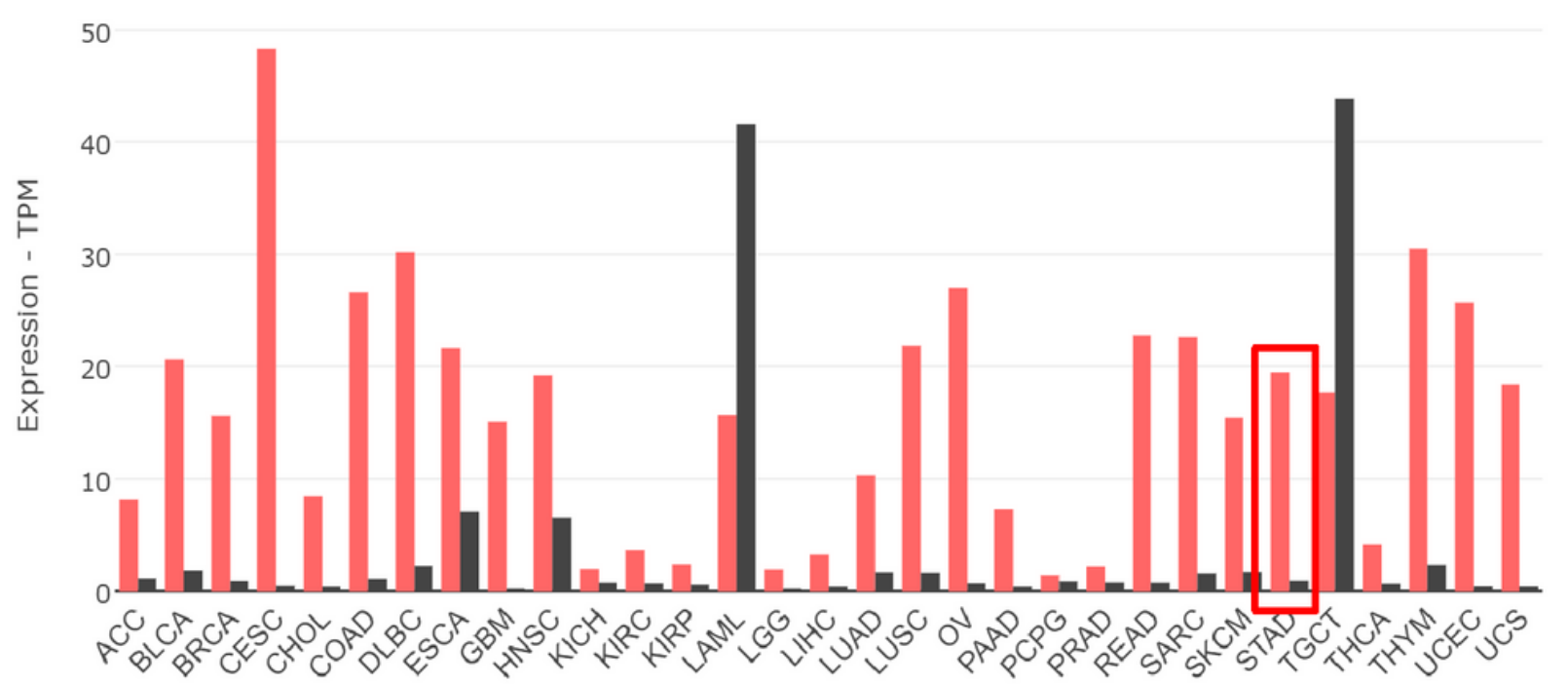

B
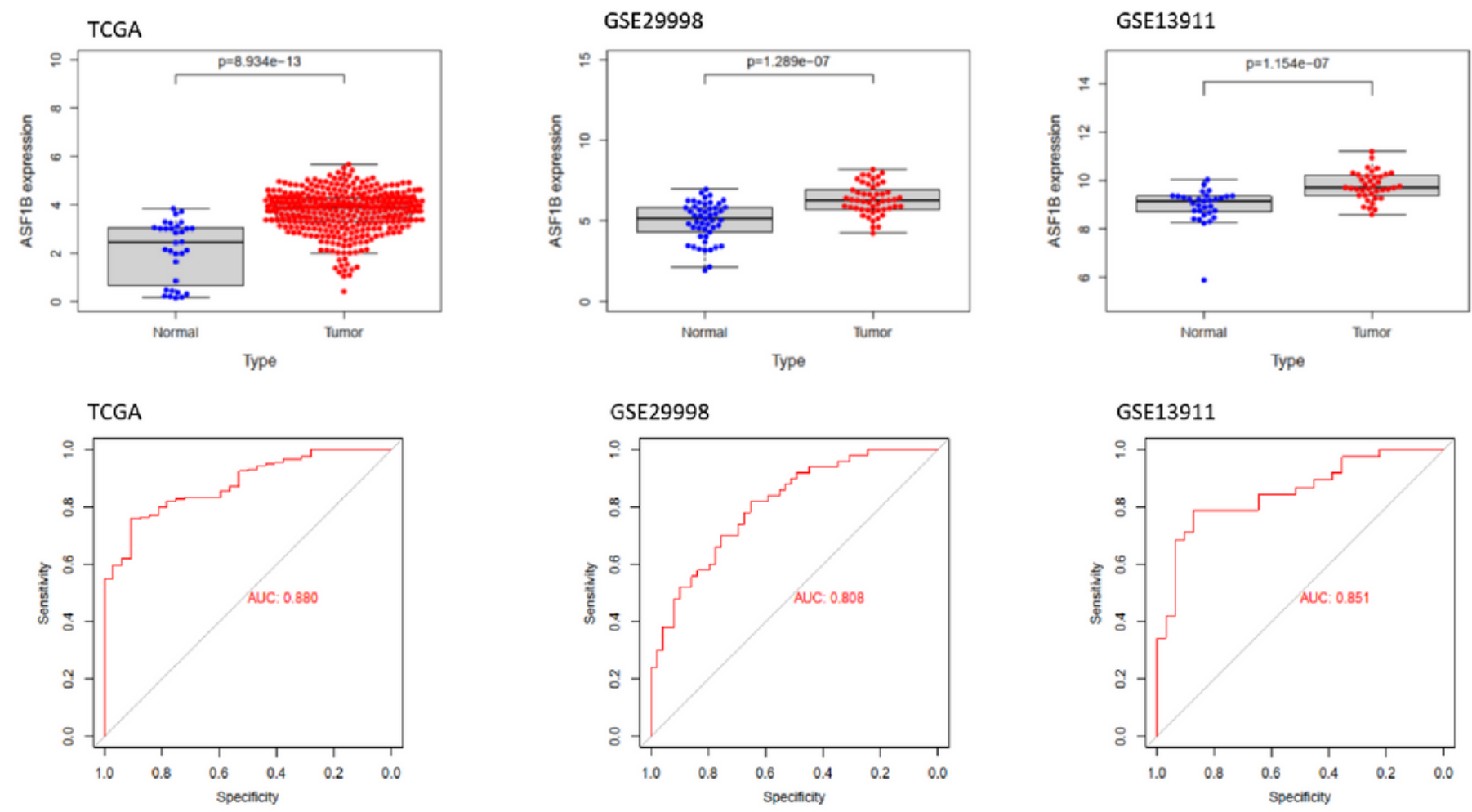

Figure 1

The analysis of ASF1B expression profile. (A) Expression of ASF1B in various cancers (GEPIA 2.0). (B) The mRNA expression of ASF1B is up-regulated in TCGA, GSE29998 and GSE13911; ROC curve of ASF1B in predicting tumorigenesis. 
A

TCGA OS
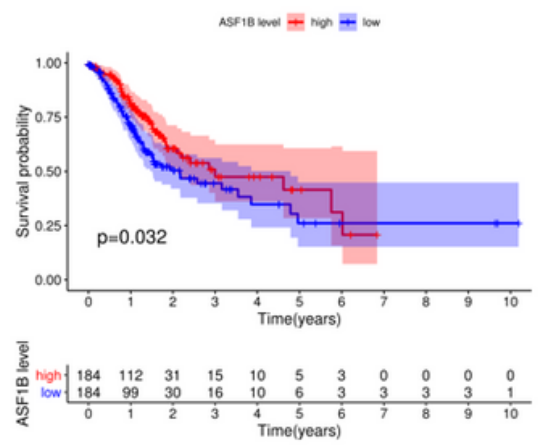

TCGA PFS

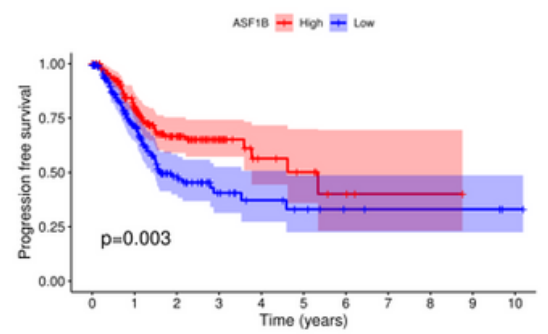

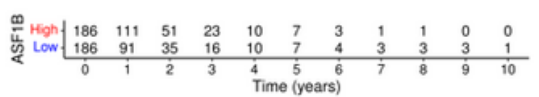

GSE84437 OS

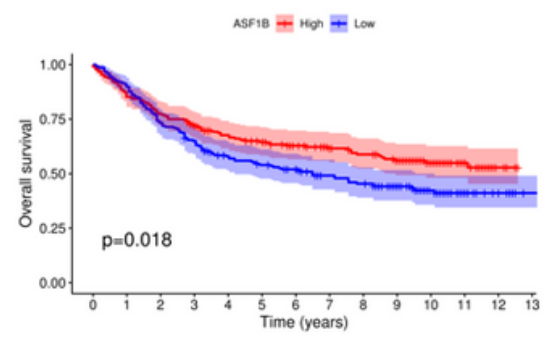

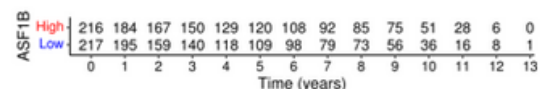

B

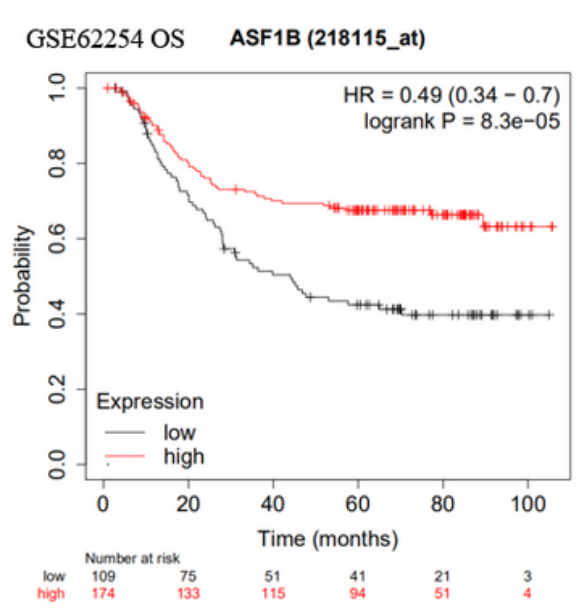

C GSE84437

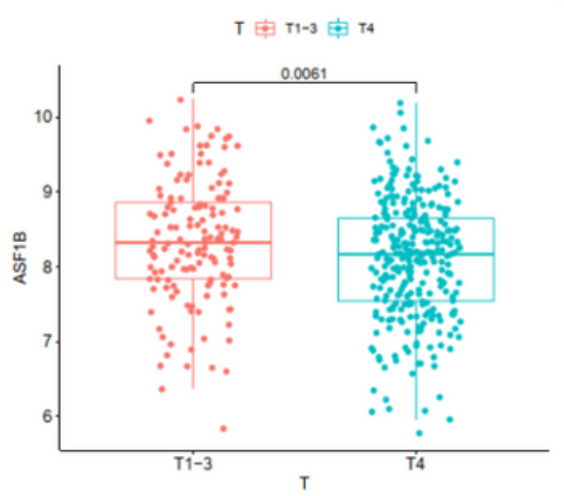

\section{Figure 2}

The clinical correlation and prognosis analysis of ASF1B. (A) Kaplan-Meier curve of ASF1B expression in GSE84437 and TCGA dataset; (B) ASF1B expression in GSE84437 from Kaplan-Meier Plotter (http://kmplot.com/) (C) Comparison of ASF1B expression in T stage (GSE84437). 

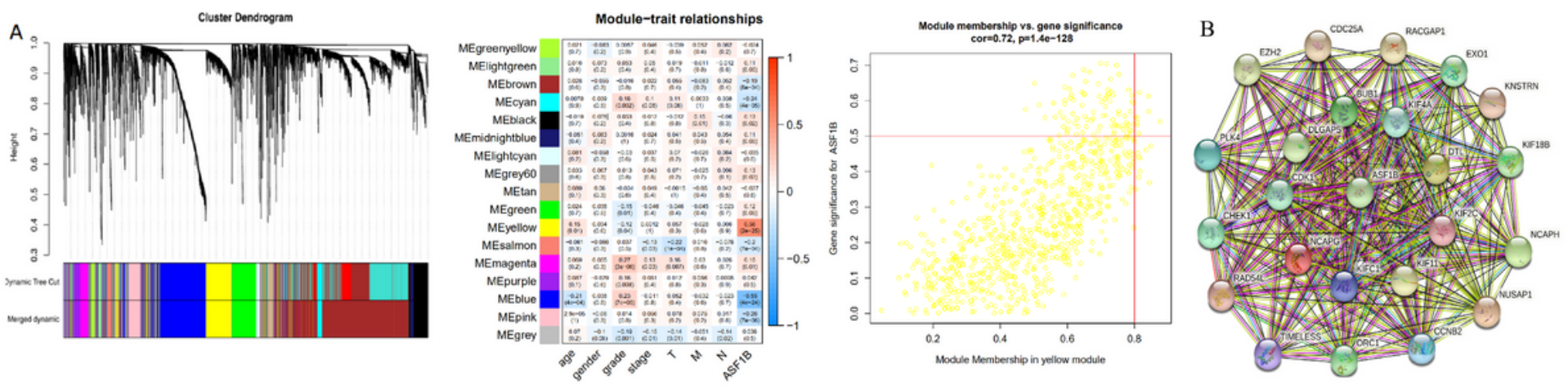

$\mathrm{C}$
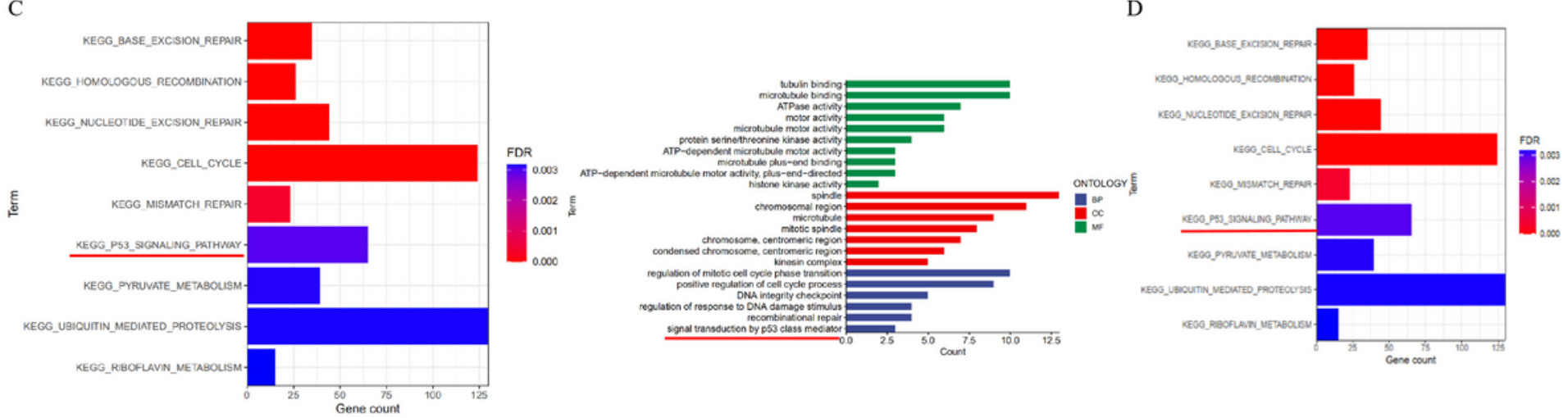

Figure 3

GSEA enrichment analysis and WGCNA construction PPI, GO and KEGG. (A) The cluster dendrogram of coexpression network modules were ordered by a hierarchical clustering of genes based on the 1-TOM matrix. Each module was assigned to different colors. Each row corresponds to a color module and column corresponds to a clinical trait. Each cell contains the corresponding correlation and P-value. The yellow module genes were used to construct the PPI network. (B) GO analysis of module genes; (B) KEGG analysis of module genes. (C)Results of GSEA enrichment analysis in ASF1B high expression group. 
A

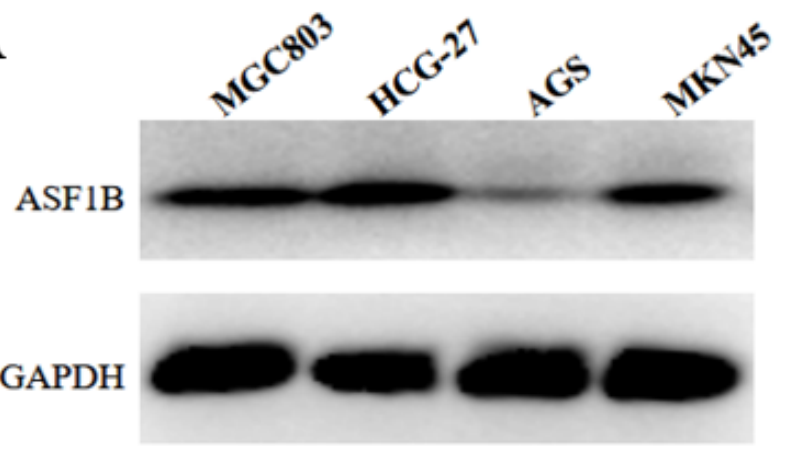

$\mathrm{B}$
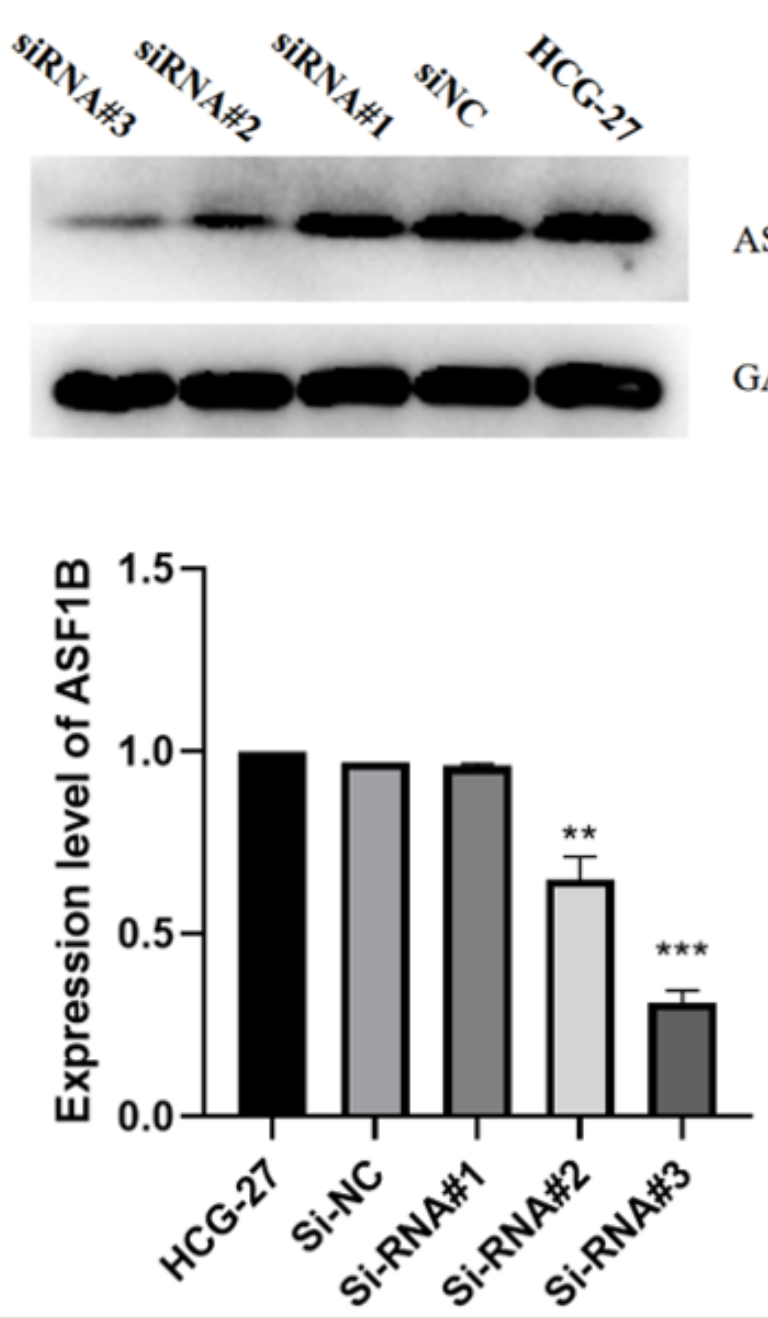

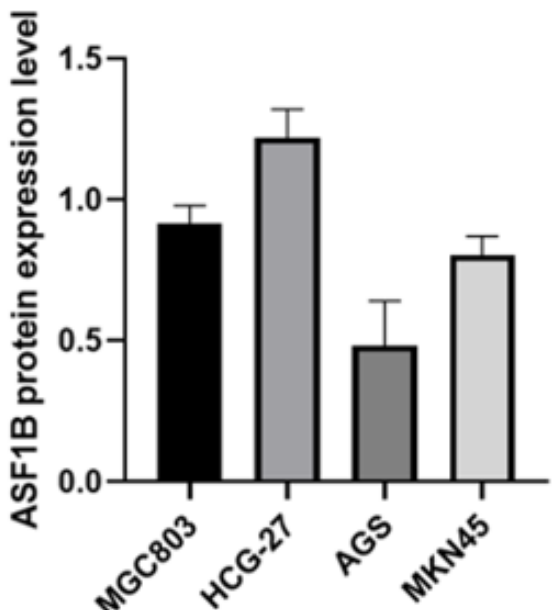

ASF1B

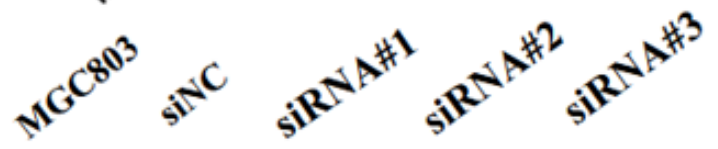

GAPDH

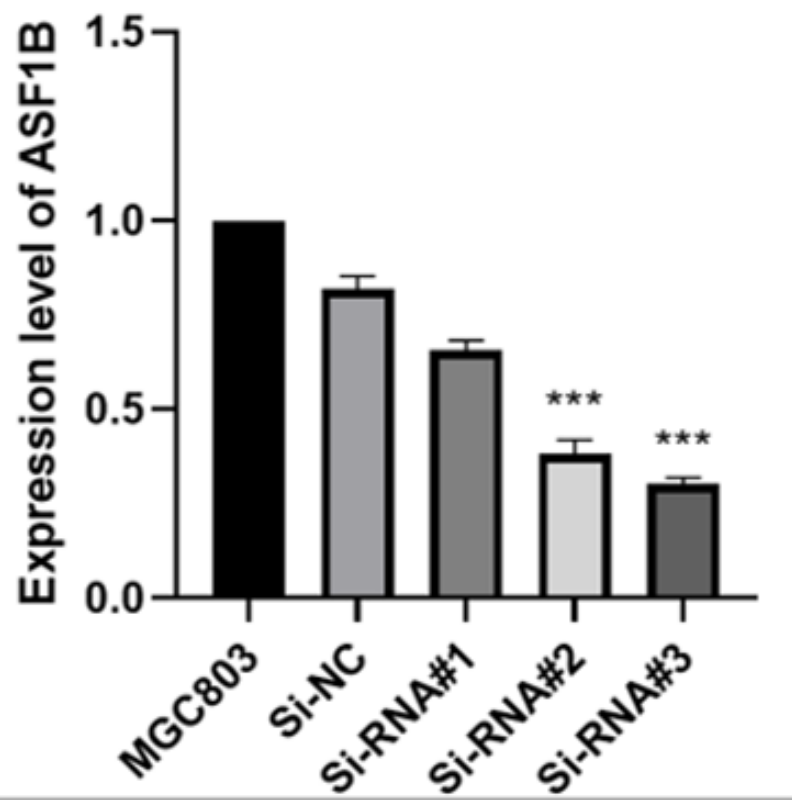

Figure 4

Expression of ASF1B in gastric cancer cell line. (A) the expression of ASF1B protein in four cell lines was detected by western blot. (B) the transfection efficiency of siRNA was detected by western blot. ${ }^{*} \mathrm{P}<0.001 \rrbracket$ $* * P<0.01 \rrbracket * P<0.05$. 
A

MGC803

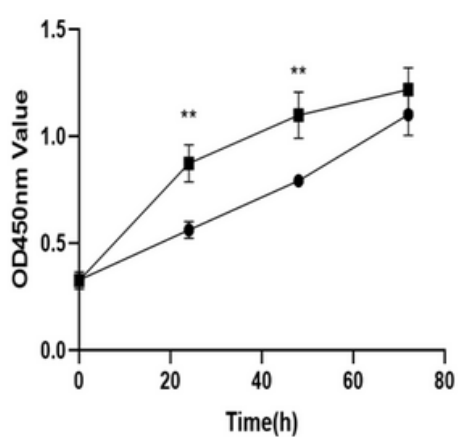

B

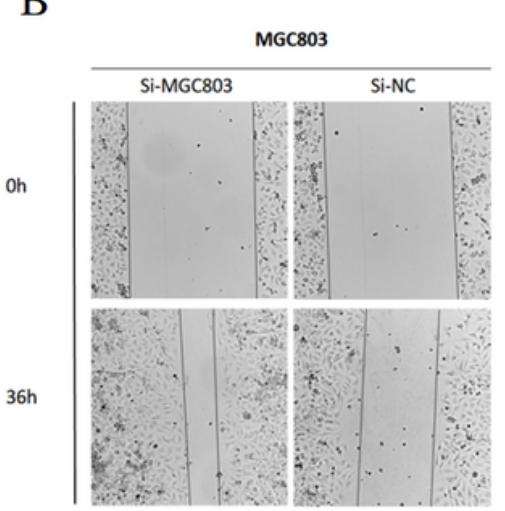

- Si-NC

- Si-ASF1B

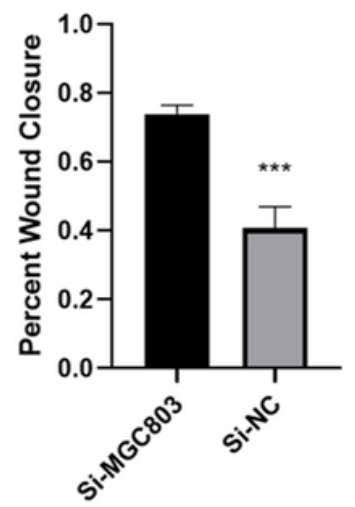

HGC-27

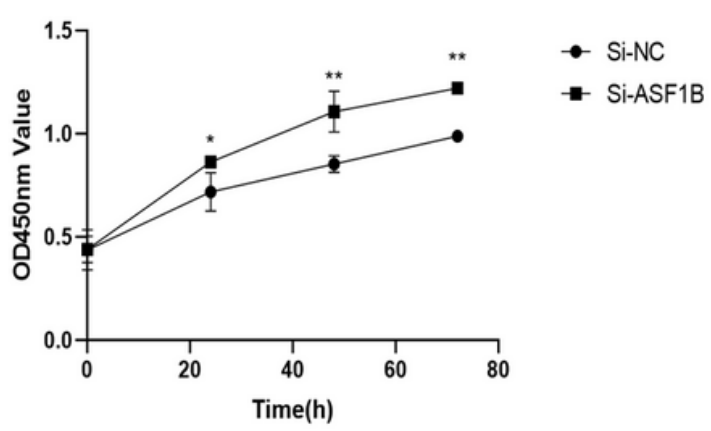

Oh

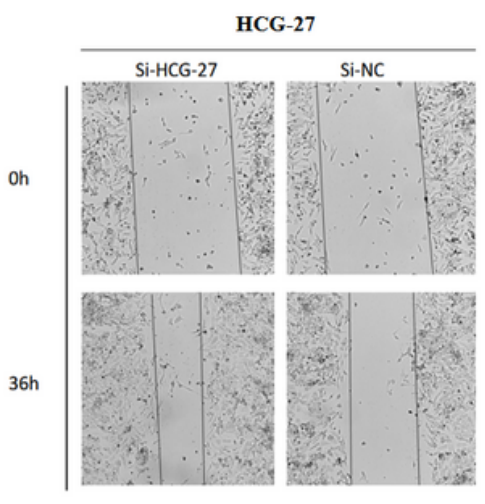

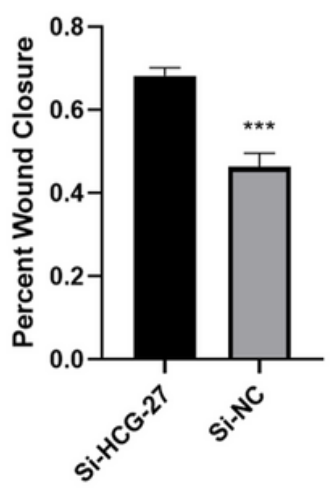

\section{Figure 5}

Effect of ASF1B on proliferation and migration of gastric cancer cells. (A) the activity of cell proliferation was measured by CCK-8 colorimetry. (B) Experimental study of wound healing to study the role of ASF1B in the migration of MGC803 and HCG-27 cells. 
A

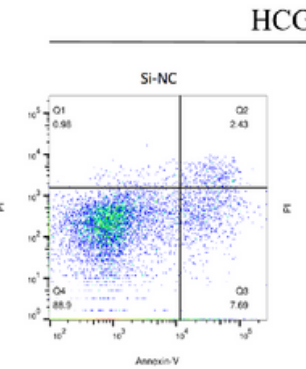

HCG-27
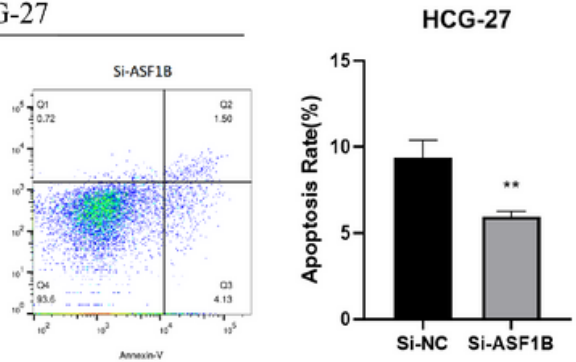

B
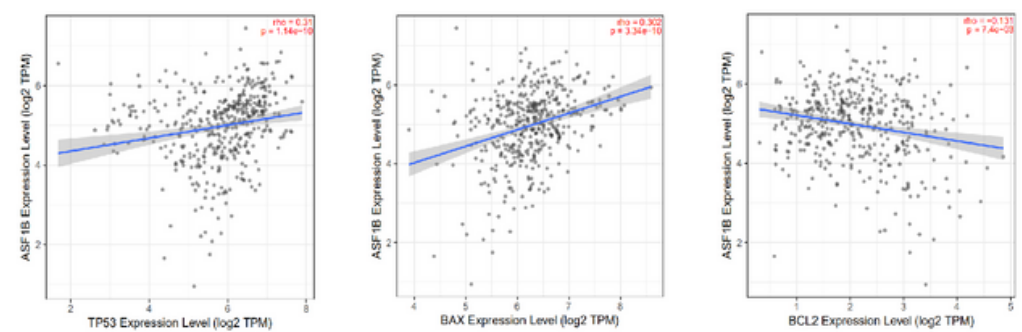

MGC803
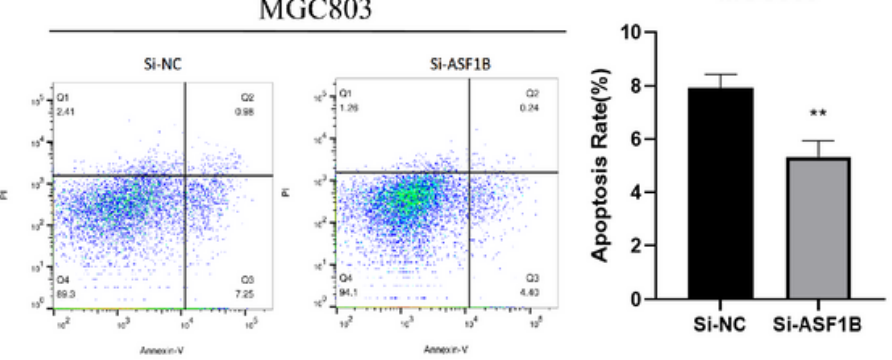

C
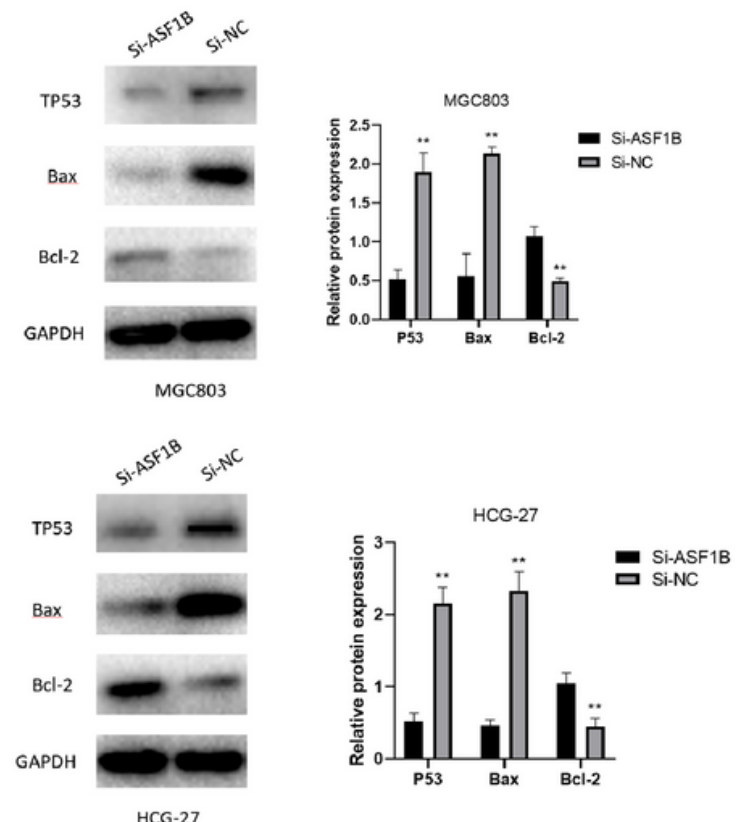

Figure 6

ASF1B regulates apoptosis of gastric cancer cells. (A) Apoptosis was detected by flow cytometry. (B) Correlation between ASF1B and key genes of the p53 apoptosis pathway. (C) Detection of p53 apoptosisrelated proteins in MGC803 and HCG-27 cells by western blot. 